\title{
Shared Driving Control between Human and Autonomous Driving System via Conflict resolution using Non-Cooperative Game Theory
}

\author{
Shriram C Jugade $^{1}$, Alessandro C Victorino ${ }^{2}$, Véronique B Cherfaoui ${ }^{1}$
}

\begin{abstract}
Shared driving control between human driver and autonomous driving system (AutoSys) is very significant with respect to its contribution to the field of ADAS. In this paper, we present a shared driving methodology through the fusion of the individual driving inputs of human driver and AutoSys, which computes the final driving input for the vehicle. This is achieved through the conflict resolution between the two drivers using non-cooperative game theory. The methodology of the fusion process is based on some features like driving decision admissibility (related to collision risk assessment), future predictions of driving profiles, individual driving intentions comparison (based on a similarity measure method) that are described in the article. A two player non-cooperative game is defined incorporating the driving admissibility and intentions. Conflict resolution is achieved through an optimal bargaining solution given by Nash Equilibrium. The validation is carried out on a driving simulator integrated with the softwares like IPG CarMaker and Simulink. The results for various driving scenarios are presented.
\end{abstract}

Index Terms-Shared driving, ADAS, non-cooperative game, Nash equilibrium, conflict resolution, human driving behavior, autonomous driving system, intelligent vehicles

\section{INTRODUCTION}

The field of ADAS has been continuously evolving for the better and safer driving experience. The key objective for the development of ADAS functionalities is to assist human driver. Some of the major ADAS functionalities include emergency braking system, anti-lock braking system, lane departure warning/prevention, lane change assist etc. Currently, the road map for the future developments is targeted to have fully autonomous/self-driving vehicles. Different levels of autonomy, according to the American SAE J3016 standard, (L0 for Manual Driving to L5 for full autonomous driving with no human driving controls) are designed to help with a consistent and effective development of fully autonomous vehicles. Most of the research is focused in the development of L4 level of autonomy (full autonomy with human driving controls). Human drivers are still going to play an important part from an overall performance aspect. Hence, one important issue still exist i.e. How will the

This project has received funding from the European Commission under the H2020 Grant agreement ITEAM No. 675999 and by LABEX MS2T, ROBOTEX

${ }^{1}$ Sorbonne universités, Université de Technologie de Compiègne, CNRS, Heudiasyc UMR 7253, CS 60319; 60203 Compigne Cedex, France. Email: shriram.jugade/veronique.cherfaoui@hds.utc.fr

${ }^{2}$ Department of Mechanical Engineering, Universidade Federal de Minas Gerais (UFMG), Av Antonio Carlos 6627, Belo Horizonte, Minas Gerais, Brazil. Email: avictorino@ufmg.br

Validation of the presented shared control strategy was carried out on the test rig at Jaguar Land Rover, National Automotive Innovation Centre, Coventry UK. transition between manual driving mode and autonomous driving mode take place? Also, the autonomous vehicles encounters various driving issues and need to be resolved with the help of human driver. One of the approach to address these issues is shared driving control.

There have been several approaches for the shared driving control in the past researches. In [1], shared control is achieved through the design of safe trajectories based on the preferred velocity of the human driver. The lateral dynamics of the vehicle play an important safety role in various driving scenarios. To assist the human driver in the vehicle steering, shared steering control is developed using haptic feedback ([2], [3], [4], [8]). The haptic feedback creates an intervention in the human control actions for the necessary correction which also prevents the human driver to express his driving actions independently. Shared driving can be applied to both pedal and steering inputs in the process of generalization as shown in [11]. In some cases, the shared driving is focused on specific situations like road departure prevention ([14]), braking scenarios in the presence of pedestrians ([15]) etc.

There have been various methods presented in the past researches to develop shared control. Since both human driver and autonomous driving system (AutoSys) are involved in the shared control, differential game approach is used ([5]). In these methods, the human driver is expected to behave cooperatively with the AutoSys. There can be various conflict situations arising if the human driver behavior is independent. Similarly, different control approaches have been used such as model predictive control and its variants ([10], [12]), fuzzy control ([13]). These approaches have a high dependency on the vehicle model (linear/nonlinear) for the decision making.

Human interaction with AutoSys is modeled in [6] to improve the performance. Shared driving is also viewed as an multi-agent system. In [7], two human drivers have been included to explore the concept. Shared driving approaches are subjective in nature. It can also be realized through the traded/switching control between human and AutoSys ([9]).

In this paper, we present a shared driving methodology through the fusion of the individual driving inputs produced from human and AutoSys (Figure 1) with the following expectations:

- Application to both longitudinal and lateral driving

- No dependency on the vehicle model

- Decision making approach instead of a control approach

- Application to multiple driving scenarios

- Allowing the human driver to behave independently i.e. no intervention through haptic feedback 
The rest of the paper is organized as follows: Section II presents the framework and model architecture of the shared driving control methodology along with the description of various sub-systems and their respective functionalities. Section III presents the development of the fusion system with respect to the model architecture. Section IV presents the integrated closed loop validation strategy, setup and results. Section V presents the conclusion based on the methodology and results.

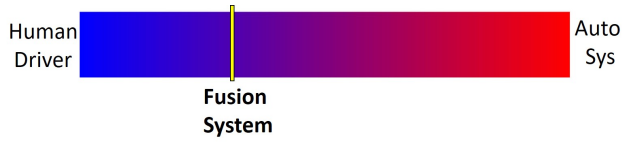

Fig. 1. The presented methodology creates a continuous control authority scale between the human driver and AutoSys. An example of control authority division using fusion system.

\section{Shared Driving Strategy: Modeling ASPECTS}

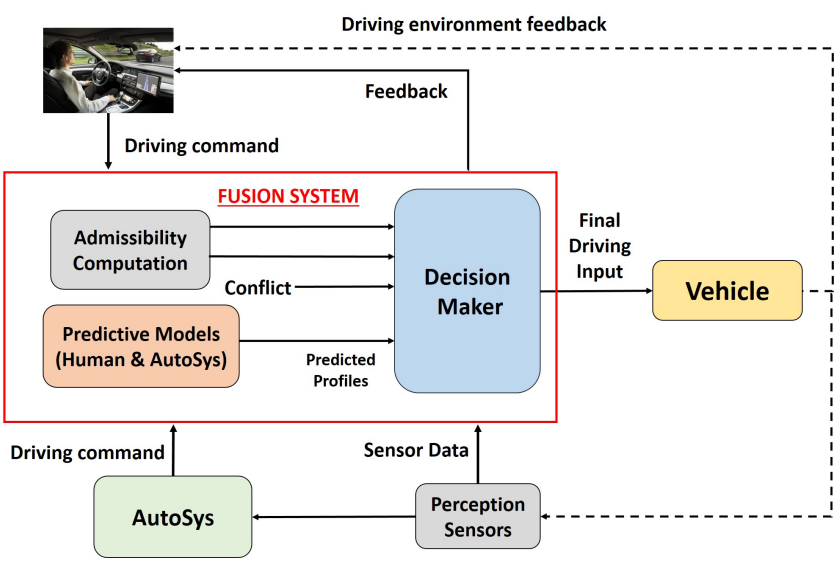

Fig. 2. Global Methodology for Shared Driving

Fig. 2 presents the global methodology for the shared driving control using fusion system. Fusion system individually doesn't need the driving intelligence. It relies on the driving inputs from human and intelligent system. From the point of view of vehicle control, the fusion system is considered to be the high level controller. A separate low level controller is required for the tracking purpose. Hence, the presented fusion methodology is independent of vehicle dynamics/model. The fusion system consists of various subsystems each performing a specific function, as described in the next sub-sections.

\section{A. Driving Decision Admissibility}

The individual driving inputs need to be assessed for their admissibility/acceptability with respect to the collision risk, road departure prevention etc using vehicle and environment states. The environmental state is obtained through the occupancy grid map formed from the LIDAR and Camera sensor data. The sensor measurement noise creates uncertainty about the environment which is propagated in the admissibility. Hence, instead of assessing the driving input in the binary form (admissible/inadmissible), a degree of belief of decision admissibility $(0-100 \%)$ is calculated using belief function theory. This uncertainty can also be seen as the level of confidence in the decision with respect to its admissibility. The detailed methodology developed in our past research is presented in [16].

\section{B. Driving Behavior Prediction}

For blending the current driving inputs, it is important to consider the driving intention. The final driving command is computed by looking into the future driving behavior of human and intelligent system. For this purpose, predictive models based on neural network are used. The model predicts the future behavior over a time horizon of 2-4 secs by considering the current and past states of environment, vehicle and driver. The model outputs the future profiles of intended vehicle speed and steering wheel angle. Separate model with the same architecture were trained using the driving data of both human and intelligent system. The detailed methodology developed in our past research is presented in [17].

\section{Decision Maker}

The driving decision admissibility and the predicted driving behaviors are passed to the decision maker for the computation of final driving command. This sub-function is the main contribution of the this paper. This algorithm is based on a non-cooperative game theory to find a bargaining solution between the human and intelligent system for conflict resolution, as explained in details in the next sections.

\section{DECISION MAKING}

This section presents the shared control strategy used by the decision maker for the computing the final driving command.

\section{A. Fusion via Conflict Resolution}

Human and AutoSys have different control strategies i.e. their intended state trajectories of the plant are different. This can be viewed as a conflict between human and AutoSys. The fusion system needs to quantify it and consider this conflict for its function. Hence, we define conflict at a given time instant as the difference between individual control inputs: if $u_{1}(t)$ and $u_{2}(t)$ are the control inputs of human and AutoSys respectively then, the conflict is given as:

$$
\text { Conflict }(t)=u_{1}(t)-u_{2}(t)
$$

The overall system consisting of human, AutoSys, fusion system and the vehicle is a closed loop. Hence, given a nonzero initial conflict, the goal of the shared control strategy is to regulate the conflict state value by finding a bargaining solution (final driving command). 


\section{B. Driving Intention Comparison using Similarity Measure}

The conflict between the human and AutoSys can be resolved if and only if their intentions are similar. For example, consider that the human driver intends to go straight while the AutoSys intends to turn right. In this case the driving intentions are completely different. The dissimilarity between the driving intentions may also arise because of the driving inadmissibility of one of the drivers. For example, in a given driving scenario, human may have failed to detect an obstacle but the AutoSys may have detected. In this case, the individual actions shall create dissimilarity between the driving intentions. This aspect will be reflected in the individual degrees of admissibility.

The predicted driving input profiles represent the driving intention. We define a similarity measure for comparing the profiles of human and AutoSys thus giving the degree of similarity between the individual driving intentions. The predicted profiles can be considered as input trajectories for the purpose of comparison. The shape of these trajectories defines the driving intention. The presented similarity measure gives the degree of shape similarity.

Fig. 3 show sample vehicle speed profiles (predicted) for a given braking scenario. These speed profiles can be represented by the coefficients obtained from the 2 nd order polynomial regression. Thus, the similarity of the profiles is transformed to the similarity of their respective scaled polynomial coefficients represented as Cartesian coordinates on a $2 \mathrm{D}$ plane as shown in Fig. 4.

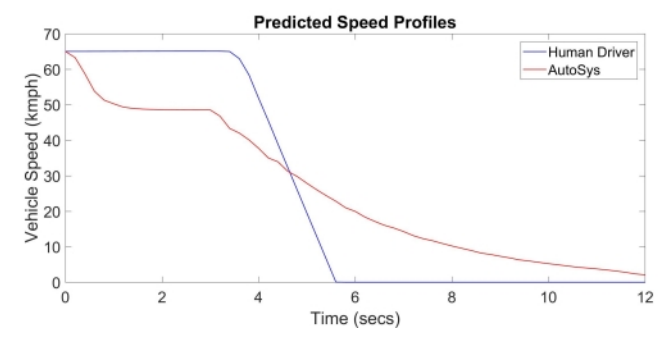

Fig. 3. Sample predicted vehicle speed profiles

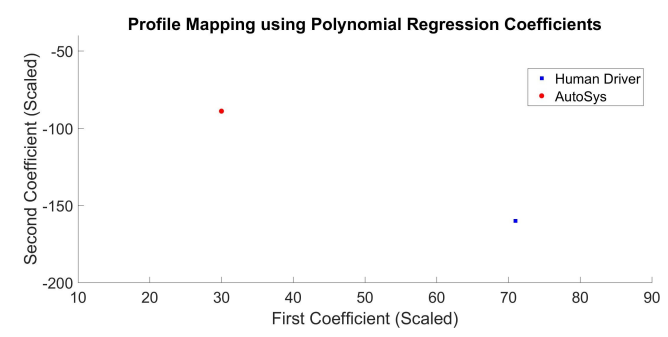

Fig. 4. Scaled coefficients of polynomial regression of vehicle speed profiles in Fig. 3

The similarity measure between the predicted trajectories is inversely proportional to the Euclidean distance between the respective points on the $2 \mathrm{D}$ plane. The similarity measure between two trajectories is given by:

$$
\operatorname{Sim}\left(T_{1}, T_{2}\right)=\frac{1}{1+\alpha * E_{d}}
$$

where $E_{d}$ is the Euclidean distance and alpha is the scaling factor (0.01). It is evident from the Fig. 4 that the similarity between the profiles (red and blue) is higher than that between the profiles (red/blue and yellow).

\section{Conflict Resolution via Non-Cooperative Game}

We define a non-cooperative game between the drivers using their respective predicted driving intentions and admissibility for the conflict resolution. The bargaining solution to this non-cooperative game forms the final driving command for the vehicle.

1) Loss Utility Function: The final driving command can be viewed as a deviation from the individual driving commands of human and AutoSys. Depending on the amount of deviation, the drivers will have some degree of acceptability/resistance towards the final driving decision.

The resistance towards final driving decision can be represented by a loss utility function. This is dependent on various factors like environmental state, driving skills, individual etc. which makes it very subjective. One approach is to consider the loss utility function completely random but it can be generalized under the assumption that the resistance and the rate of resistance increases as the deviation between the individual driving intention and the final driving intention increases. With respect to this assumption, the loss utility function is considered to be paraboloid as shown in the Fig. 5 .

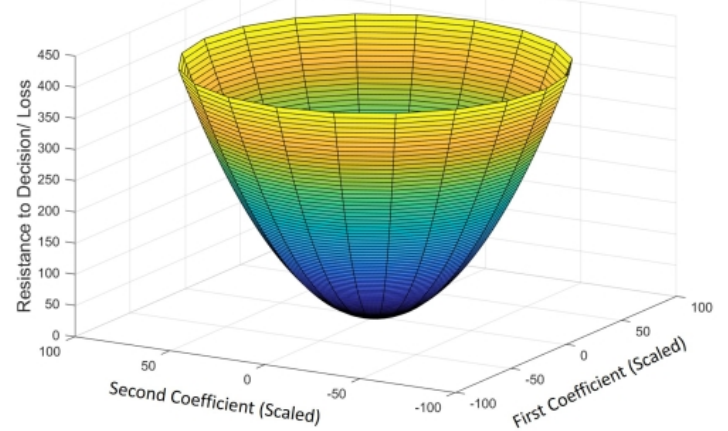

Fig. 5. Sample Loss Utility function

2) Non-Cooperative Game using Loss Utilities: Consider the Fig. 3 containing individual driving intentions (predicted) along with their respective loss utility functions. The final driving command (for e.g. intended vehicle speed) is calculated from the final driving decision profile (for e.g. intended vehicle speed profile), selected on the 2D plane (Fig. 4). The conflict between the two drivers is directly proportional to the absolute difference between the respective loss utility values. We define a non-cooperative game assuming the case where both the drivers are given a choice to select the final driving decision/intention. Hence, the tendency of both the drivers will be to select their individual driving intentions (original). 
Let $x_{1}$ and $x_{2}$ be the respective selections. Consider $L_{1}()$ and $L_{2}()$ as the loss utility functions of both the drivers. The difference in loss utility for the selection $x_{1}$ is $\delta L\left(x_{1}\right)=\left|L_{1}\left(x_{1}\right)-L_{2}\left(x_{1}\right)\right|$ and that for $x_{2}$ is $\delta L\left(x_{2}\right)=$ $\left|L_{1}\left(x_{2}\right)-L_{2}\left(x_{2}\right)\right|$. The optimal selection of the final driving decision/intention with respect to this non-cooperative game is decided by

$$
\min \left(\delta L\left(x_{1}\right), \delta L\left(x_{2}\right)\right)
$$

The conflict of the final driving decision is directly proportional to the difference in the loss utility for that decision i.e.

$$
\operatorname{Conflict}(x) \propto \delta L(x)
$$

Hence, winning the non-cooperative game is equivalent to minimizing or resolving the conflict. Given the conditions of the game, the strategy of each player would be to select the Nash equilibrium to maximize the chances of winning i.e. the Nash equilibrium is the bargaining solution between the two drivers ([17]). In this case, the Nash equilibrium is the driving decision corresponding to the intersection of the loss utility functions of the drivers.

The predicted driving decision profile (for e.g. predicted vehicle speed profile) is represented as a point on the $2 \mathrm{D}$ Cartesian coordinate plane. Given the predicted decision profile of both the drivers, the Nash equilibrium solution will lie on the line segment joining these points. Hence, the projection of the loss utility function (of both drivers) onto the $2 \mathrm{D}$ plane (passing through this line segment) is sufficient for finding the Nash equilibrium solution. An example is shown in Fig. 6.

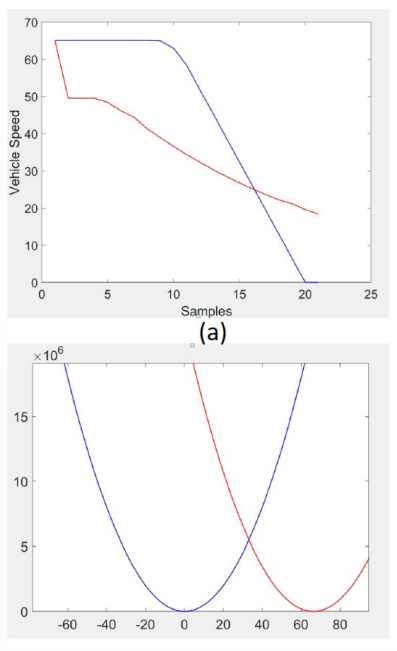

(c)

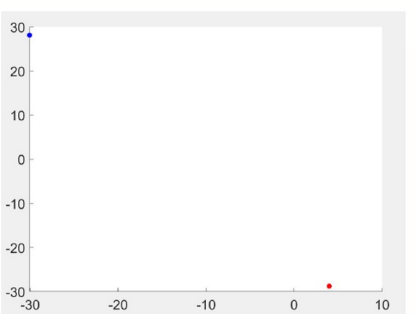

(b)

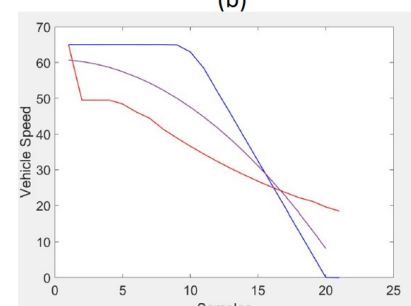

(d)
Fig. 6. Finding Nash Equilibrium. (a) The predicted speed profiles (intended) of human driver (Blue) and AutoSys (Red), (b) Mapping the profiles on the 2D plane using polynomial regression coefficients, (c) Loss utility functions (projections) with respect to the line segment and the Nash Equilibrium (intersection of the functions), (d) The final driving decision (vehicle speed) profile corresponding to the Nash Equilibrium
3) Incorporating Decision Admissibility: The noncooperative game can also be viewed as a negotiation. The bargaining solution depends on the ability of each player to negotiate. Since the loss utility function represents the resistance to change, it can also be viewed from the point of view of willingness for negotiation. For e.g. if the shape of the utility function is large, then the resistance to change is lesser i.e. the player/driver is more willing to negotiate for the change in driving decision. This can be correlated to the degree of driving decision admissibility i.e. lower the degree of admissibility, lesser is the resistance to change. Thus, the shape (steepness) of the paraboloid (loss function) is correlated to the degree of driving decision admissibility.

Consider the example shown in Fig. 6. In this case, we will assume the degree of decision admissibility of one of the driver to be lower. The fusion of the driving inputs in such case is shown in Fig. 7. The individual driving decision with higher degree of admissibility is given more preference during the fusion process.

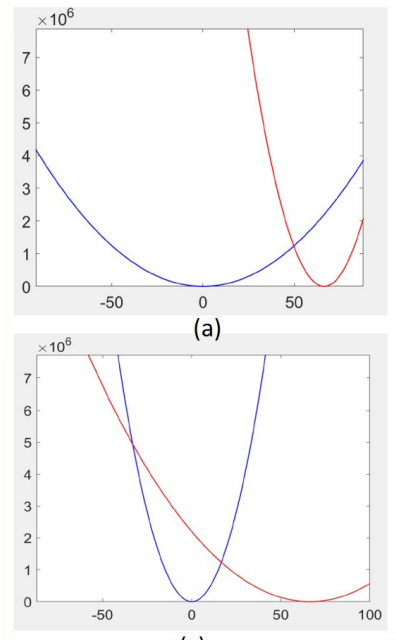

(c)
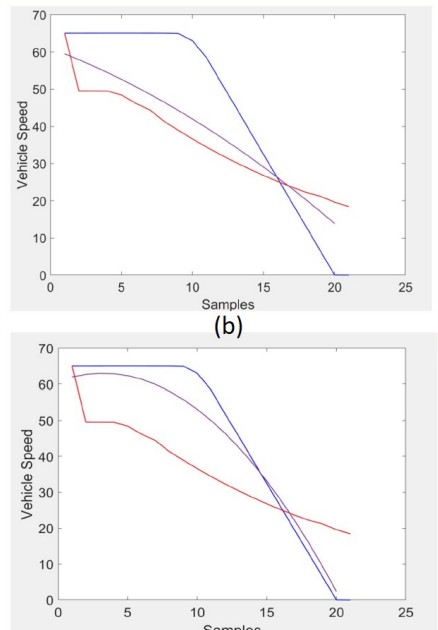

(d)
Fig. 7. Effect of decision admissibility on the Nash Equilibrium (a) Loss utility functions (projections) for both human driver (Blue) and AutoSys (Red). The loss utility function of the human driver is less steep due to the lower admissibility (b) Predicted speed profiles for human driver (Blue) and AutoSys (Red) along with final speed profile corresponding to Nash equilibrium, (c) Loss utility functions (projections) for both human driver (Blue) and AutoSys (Red). The loss utility function of the AutoSys is less steep due to the lower admissibility, (d) Predicted speed profiles for human driver (Blue) and AutoSys (Red) along with final speed profile corresponding to Nash equilibrium.

Similarly, the above fusion methodology can be extended and applied to the steering wheel angle profiles (predicted). Thus, the final fusion output is given in the vector form including final intended vehicle speed and steering wheel angle.

4) Incorporating Individual Driving Intentions: The predictive models provide the speed and steering angle profiles for both human driver and AutoSys which are mapped to a 2D plane using quadratic polynomial regression coefficients (scaled). These mappings are considered as the quantification of individual driving intentions. Given the driving decision 
admissibility of both human driver and AutoSys are high $(\geq 70)$, the fusion methodology mentioned in the previous subsections is compatible if and only if the driving intentions are similar. Hence, a lower threshold with respect to the similarity measure is considered for matching the driving intentions. If the similarity value is lower than the threshold, then the driving intentions are considered to be different and the fusion is not compatible. In such a case, the fusion system follows the human driving inputs i.e. becomes human centric. The lower threshold is tunable and considered to be $60 \%$ for the validation purposes.

5) Feedback to the Human Driver: The final driving input (pedals,steering) may be different than that of the human driver. This difference may confuse the driver or create a panic situation. Hence, it is necessary to inform the human driver about the driving input deviation. The parameters fed back to the human driver are driving decision admissibility, intended speed of human driver, intended speed of fusion system (actual vehicle speed), steering wheel angle inputs of human driver and fusion system.

\section{EXPERIMENTAL VALIDATION}

The validation of the shared driving is carried out over both longitudinal and lateral navigation control. The driving scenarios are categorized with respect to different aspects as shown in the table below. The categorization of the decision admissibility (High/Low) is only for the explanation purpose but is considered continuous during calculations.

\begin{tabular}{|l|l|l|l|}
\hline Type & $\begin{array}{l}\text { Human Driver } \\
\text { Admissibility }\end{array}$ & $\begin{array}{l}\text { Auto Sys } \\
\text { Admissibility }\end{array}$ & $\begin{array}{l}\text { Driving } \\
\text { Intentions }\end{array}$ \\
\hline A & high & high & match \\
\hline B & high & high & mismatch \\
\hline C & high & low & mismatch \\
\hline D & low & high & mismatch \\
\hline
\end{tabular}

\section{A. Driving Scenarios}

For the purpose of validation, driving scenarios for the categories A, B, C and D are designed as shown in Figures $8,10,11,12$ respectively.

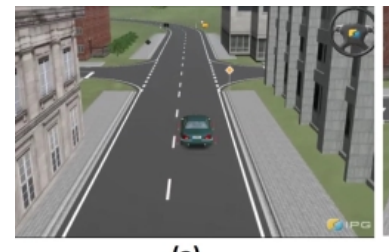

(a)

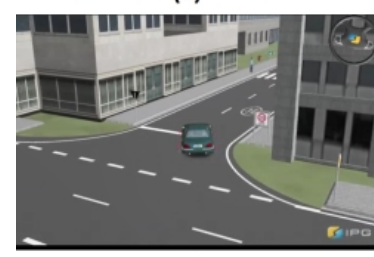

(c)

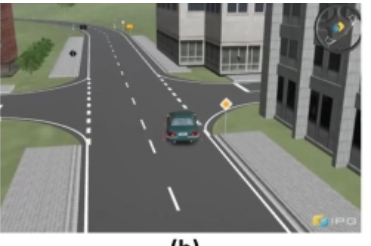

(b)

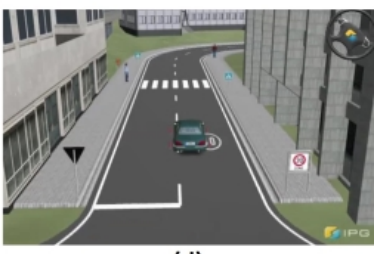

(d)
Fig. 8. Driving Scenario Type A: Sharp turn. The driving intentions of human driver and autosys match but the nature of vehicle trajectory differs. Individual driving inputs are admissible.

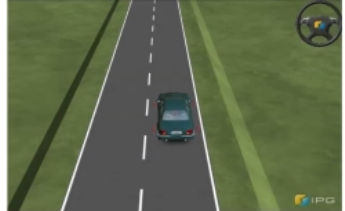

(a)

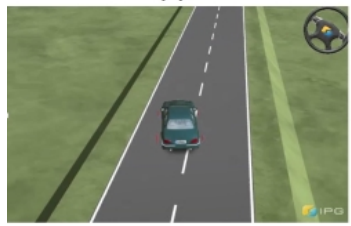

(c)

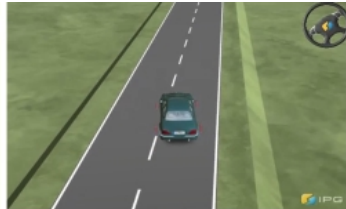

(b)

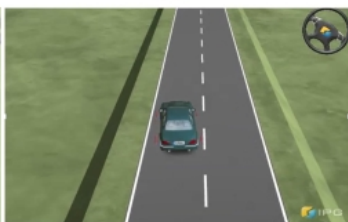

(d)
Fig. 9. Driving Scenario 2 Type A: Lane Change. The driving intentions of human driver and autosys match but the nature of vehicle trajectory differs. Individual driving inputs are admissible.

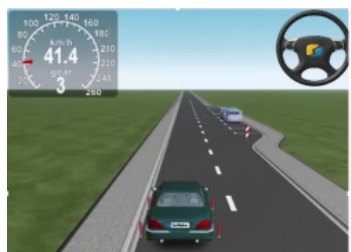

(a)

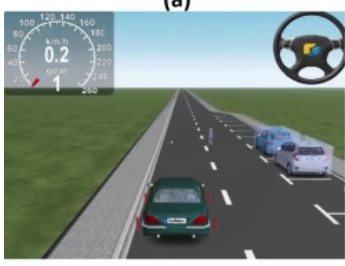

(c)

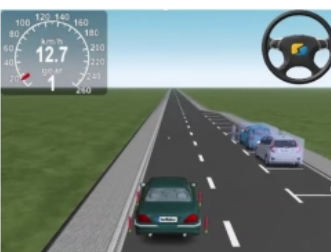

(b)

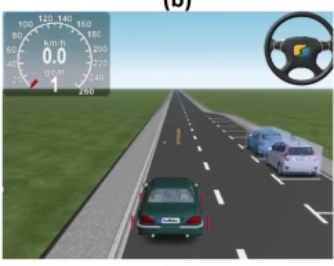

(d)
Fig. 10. Driving Scenario Type B: Target Vehicle encounters a rolling ball but the child is not visible. Human driver decelerates the vehicle to avoid probable collision, while the Auto Sys continues with the same speed. Individual driving inputs are admissible but the driving intentions do not match.

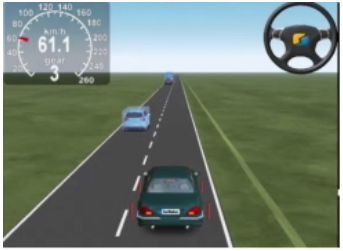

(a)

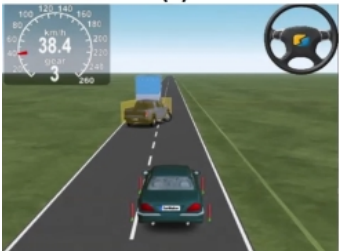

(c)

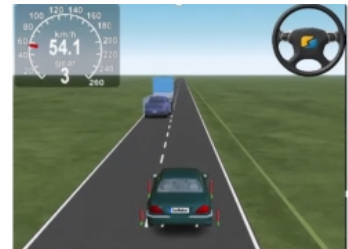

(b)

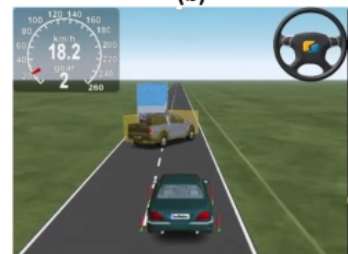

(d)
Fig. 11. Driving Scenario Type C: Stationary vehicle (in blue) suddenly sets in motion. Human driver interprets the situation pro actively and applies brakes to avoid collision (admissible decision). Auto Sys initially does not track the stationary vehicle and continues with the same speed (inadmissible decision) 


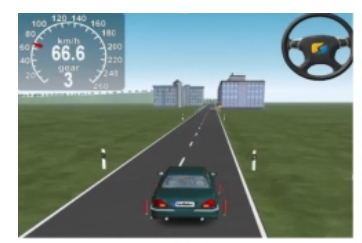

(a)

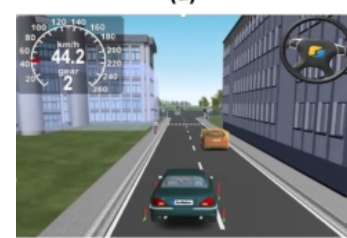

(c)

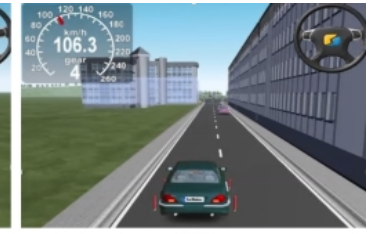

(b)

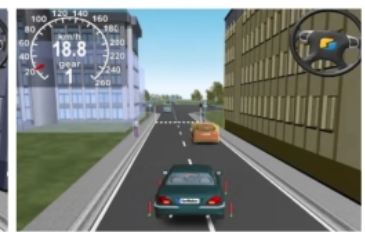

(d)
Fig. 12. Driving Scenario Type D: Highway Driving. Human driver tries to change the lane having another vehicle leading to lateral collision (inadmissible decision). Auto Sys continues to drive in the same lane (admissible decision)

\section{B. Experimental Setup}

The validation of the presented shared control strategy is carried out on a driving simulator setup integrated with the software IPG CarMaker and Simulink. The integration schematic of the setup is shown in Fig. 13. All the subsystems are modeled in Matlab/Simulink. The Simulink model receives the human inputs from the driving setup. The fusion system uses autonomous driving system inbuilt in IPG CarMaker. The block diagram of the shared driving control process implemented in the Simulink model is shown in Figure 14.

The pedal inputs from the human driver and AutoSys are converted to speed inputs termed as intended speed. The intended speed profiles and the final vehicle speed profile (from fusion system) may differ. At any given time instant, the human driver or AutoSys may want to jump to the final vehicle speed profile from their respective intended speed profile. For such cases, the human driver or AutoSys can stop giving pedal inputs momentarily. This is an add-on provision in the test setup. Therefore, transients can be expected in the intended speed profiles. Since these individual speed profiles are not directly executed on the vehicle but just serve as inputs for the fusion, they can be considered realistic.

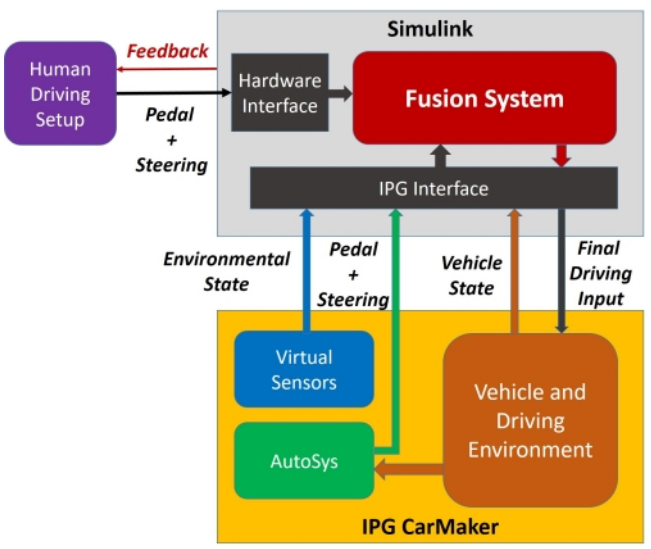

Fig. 13. Shared Control Strategy Validation Setup

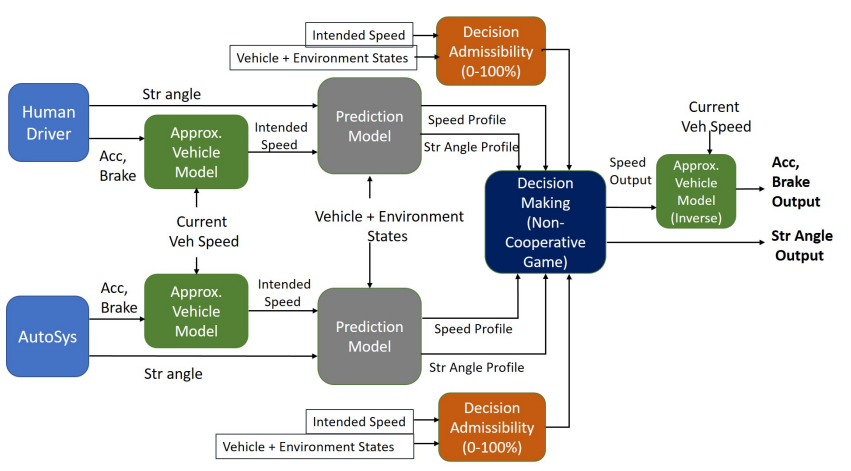

Fig. 14. Block Diagram of Shared Driving Control Implementation

\section{Experimental Results and Analysis}

The driving inputs fusion is done with respect to individual decision admissibilities and driving intention similarity. With respect to the driving safety, the decision admissibility has higher priority than the driving intentions.

The validation results related to Scenario type A (Fig. 8) are shown in Fig. 15, 16 and 17. Both the driving inputs are admissible throughout the scenario and the fusion is done with respect to the individual driving intentions during different phases of the scenario. Till $\mathrm{T}=25 \mathrm{secs}$, the conflict value is very low. At $\mathrm{T} \geq 25$ secs, the conflict starts to increase and again decreases at $\mathrm{T}=31$ secs. The fusion of the driving inputs takes place based on the similarity of driving intentions. Thus whenever, the difference in the driving intention. Thus, the human driving profile is refined and improved using that of AutoSys. The validation results related to Scenario 2 type A (Fig. 9) are shown in Fig. 18, 19, 20. Similar to the earlier scenario, the human driving profile is refined and improved using that of AutoSys. The fusion of the driving inputs (speed and steering wheel angle) result in the fusion of the intended vehicle trajectories.

The validation results related to Scenario type B (Fig. 10) are shown in Fig. 21 and 22. As seen in Fig. 22, the individual driving actions are admissible but the driving intentions are different (Fig. 21). Hence, the fusion system gives more preference to the human driver (human-centric). In such critical scenarios, it is always safe to follow human driver. In Fig. 21, the individual speed profiles are same till $\mathrm{T}=1 \mathrm{sec}$ and then start differing but the fusion system smoothly follows the human driver without any transients. This phenomenon is seen because of the consideration of future driving behavior predictions during decision making.

The validation results related to Scenario type C (Fig. 11) are shown in Fig. 23 and 24. In this scenario, just before $\mathrm{T}=8$ secs, the driving actions of AutoSys become inadmissible while that of human driver remain admissible as seen in Fig. 24 . Hence, the fusion is done with more inclination towards human driving actions to avoid collision. Also, the AutoSys corrects its driving action at $\mathrm{T}=8$ secs (Fig. 23).

The validation results related to Scenario type D (Fig. 12) are shown in Fig. 25, 26. Till $\mathrm{T}=7.5$ secs, the individual driving intentions are similar and the fusion takes place 
accordingly. For $\mathrm{T}=7.5$ secs to 16 secs, the individual driving intentions are dissimilar (similarity measure $\leq 60 \%$ ) and hence, the fusion system becomes human centric and follows human driver. From $T=7.5$ secs to 16 secs, the speed profiles of fusion system and human driver. The fusion is dependent on the individual driving decision admissibilities. Hence, at $\mathrm{T}=16$ secs, the vehicle speed is decreased sensing the collision risk. Thus, the fusion system smoothly brakes the vehicle. Here, the admissibility of human driving is low and the driving intentions do not match. Hence, the fusion system follows AutoSys to avoid collision.

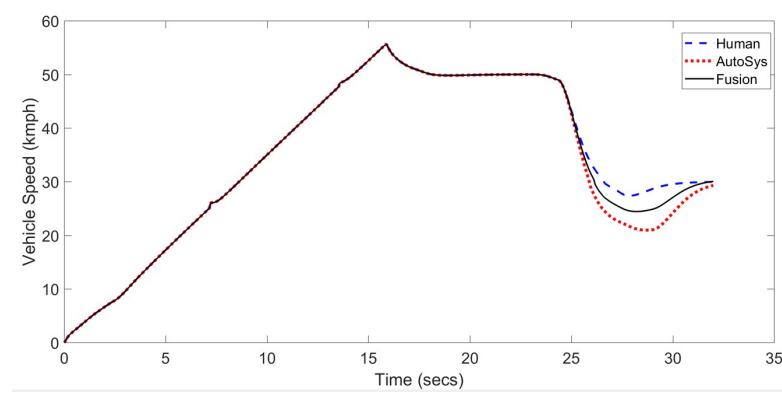

Fig. 15. Scenario Type A: Intended and Final Speed profiles

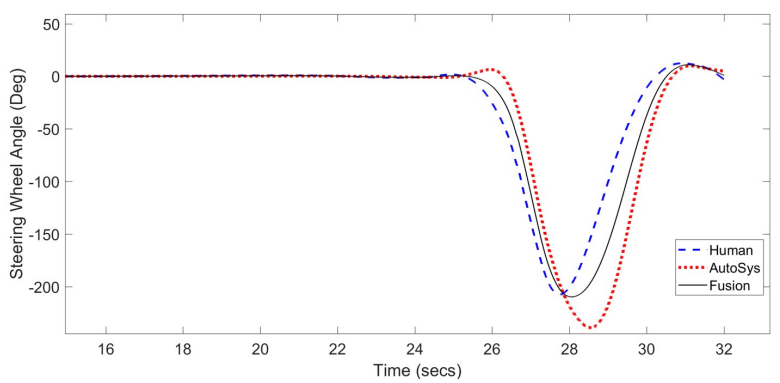

Fig. 16. Scenario Type A: Steering wheel angle profiles

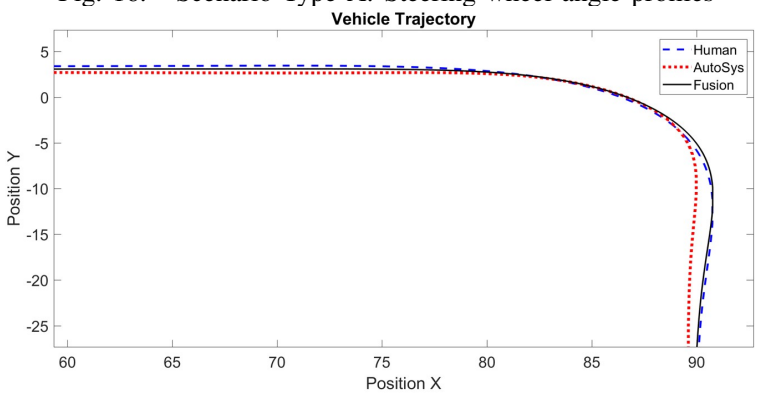

Fig. 17. Scenario Type A: Intended and Final vehicle trajectory profiles

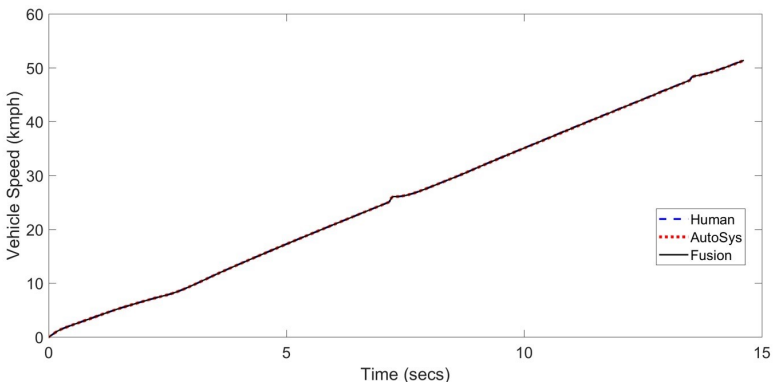

Fig. 18. Scenario 2 Type A: Intended and Final Speed profiles

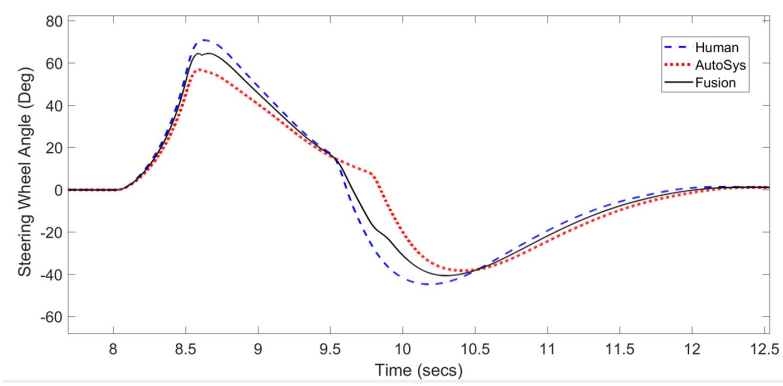

Fig. 19. Scenario 2 Type A: Steering wheel angle profiles

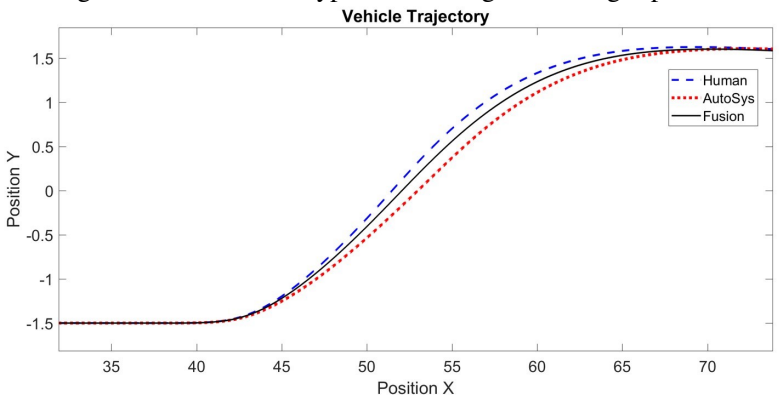

Fig. 20. Scenario 2 Type A: Intended and Final vehicle trajectory profiles

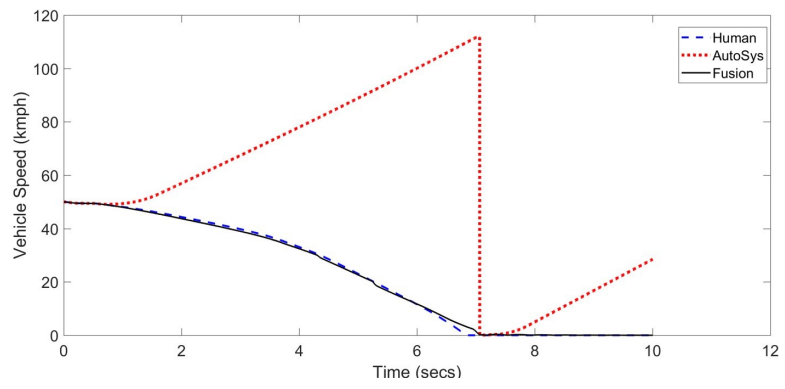

Fig. 21. Scenario Type B: Intended and Final Speed profiles

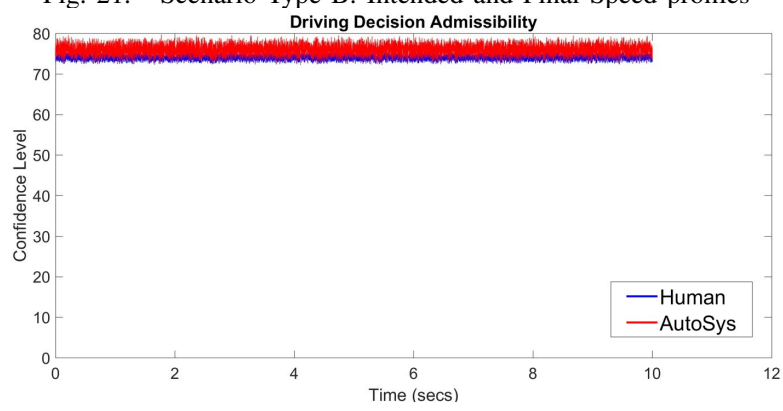

Fig. 22. Scenario Type B: Driving decision admissibility profiles

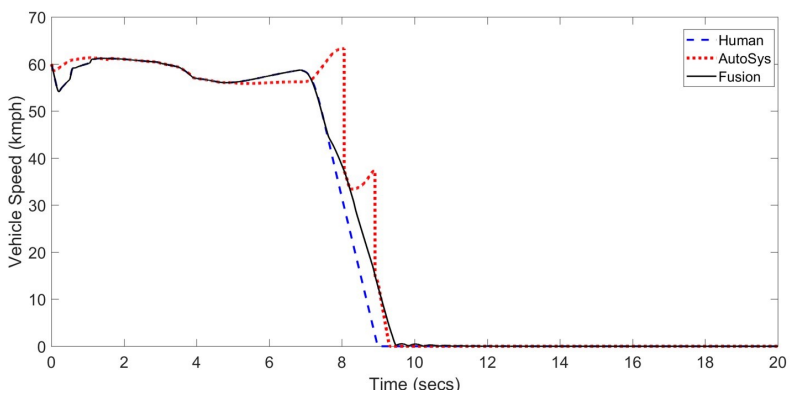

Fig. 23. Scenario Type C: Intended and Final Speed profiles 


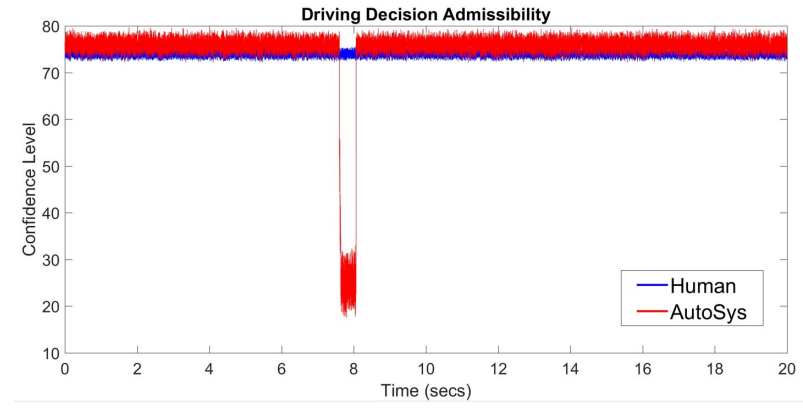

Fig. 24. Scenario Type C: Driving decision admissibility profiles

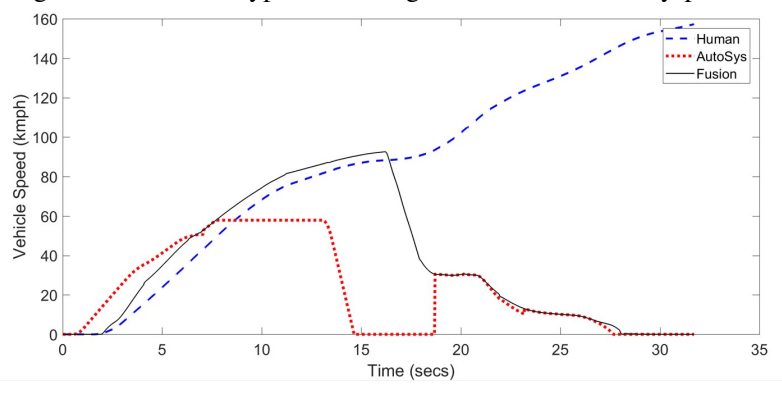

Fig. 25. Scenario Type D: Intended and Final Speed profiles

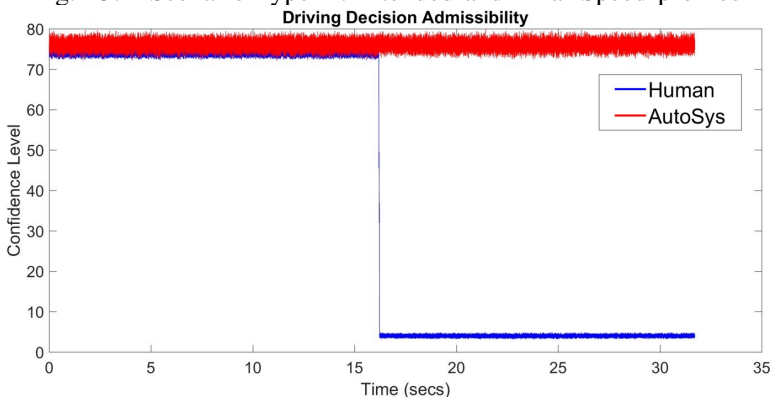

Fig. 26. Scenario Type D: Driving decision admissibility profiles

\section{CONCLUSION}

The presented strategy for decision making is based on non-cooperative game between human driver and autosys. This game minimizes the conflict between human driver and autosys through a bargaining solution. This solution acts as the final driving input for the vehicle. The parameters of this game are varied according to the individual driving decision admissibility and driving intentions. The predicted driving profiles (vehicle speed, steering wheel angle) are used to derive the driving intentions. Using the presented decision making strategy, the fusion system is not only maintains a high degree of driving decision admissibility but also minimizes the conflict between the human driver and autosys.

\section{REFERENCES}

[1] J. Alonso-Mora, P. Gohl, S. Watson, R. Siegwart and P. Beardsley, "Shared control of autonomous vehicles based on velocity space optimization," 2014 IEEE International Conference on Robotics and Automation (ICRA), Hong Kong, 2014, pp. 1639-1645. doi: 10.1109/ICRA.2014.6907071

[2] F. Borroni, M. Tanelli, A weighting approach to the sharedcontrol of lateral vehicle dynamics, IFAC-PapersOnLine, Volume 51, Issue 9, 2018, Pages 305-310, ISSN 2405-8963, https://doi.org/10.1016/j.ifacol.2018.07.050.
[3] F. Mars, M. Deroo and J. Hoc, "Analysis of Human-Machine Cooperation When Driving with Different Degrees of Haptic Shared Control," in IEEE Transactions on Haptics, vol. 7, no. 3, pp. 324-333, July-Sept. 2014. doi: 10.1109/TOH.2013.2295095

[4] P. Boehm, A. H. Ghasemi, S. O'Modhrain, P. Jayakumar, R. B. Gillespie, Architectures for Shared Control of Vehicle Steering, IFACPapersOnLine, Volume 49, Issue 19, 2016, Pages 639-644, ISSN 24058963, https://doi.org/10.1016/j.ifacol.2016.10.637.

[5] S. Mosbach, M. Flad and S. Hohmann, "Cooperative longitudinal driver assistance system based on shared control," 2017 IEEE International Conference on Systems, Man, and Cybernetics (SMC), Banff, AB, 2017, pp. 1776-1781. doi: 10.1109/SMC.2017.8122873

[6] Z. Wang, R. Zheng, T. Kaizuka and K. Nakano, "Driver-Automation Shared Control: Modeling Driver Behavior by Taking Account of Reliance on Haptic Guidance Steering," 2018 IEEE Intelligent Vehicles Symposium (IV), Changshu, 2018, pp. 144-149. doi: 10.1109/IVS.2018.8500671

[7] M. Johns et al., "Exploring shared control in automated driving," 2016 11th ACM/IEEE International Conference on HumanRobot Interaction (HRI), Christchurch, 2016, pp. 91-98. doi: 10.1109/HRI.2016.7451738

[8] D. A. Abbink, M. Mulder, E. R. Boer, "Haptic shared control: smoothly shifting control authority?," E.R. Cogn Tech Work (2012) 14: 19. https://doi.org/10.1007/s10111-011-0192-5

[9] Y. Guo, J. Liul, L. Song, H. Guo, Y. Hu and a. H. Chen, ”Hazardevaluation-based Driver-automation Switched Shared Steering Control for Intelligent Vehicles," 2018 IEEE Intelligent Vehicles Symposium (IV), Changshu, 2018, pp. 244-249. doi: 10.1109/IVS.2018.8500443

[10] C. Guo, C. Sentouh, J. Popieul and J. Hau, "MPC-based shared steering control for automated driving systems," 2017 IEEE International Conference on Systems, Man, and Cybernetics (SMC), Banff, AB, 2017, pp. 129-134. doi: 10.1109/SMC.2017.8122590

[11] J. C. F. de Winter and D. Dodou, ’Preparing drivers for dangerous situations: A critical reflection on continuous shared control," 2011 IEEE International Conference on Systems, Man, and Cybernetics, Anchorage, AK, 2011, pp. 1050-1056. doi: 10.1109/ICSMC.2011.6083813

[12] W. Schwarting, J. Alonso-Mora, L. Paull, S. Karaman and D. Rus, "Safe Nonlinear Trajectory Generation for Parallel Autonomy With a Dynamic Vehicle Model," in IEEE Transactions on Intelligent Transportation Systems, vol. 19, no. 9, pp. 2994-3008, Sept. 2018. doi: 10.1109/TITS.2017.2771351

[13] M. Li, H. Cao, X. Song, Y. Huang, J. Wang and Z. Huang, "Shared Control Driver Assistance System Based on Driving Intention and Situation Assessment," in IEEE Transactions on Industrial Informatics, vol. 14, no. 11, pp. 4982-4994, Nov. 2018. doi: 10.1109/TII.2018.2865105

[14] D. Katzourakis et al., "Shared control for road departure prevention," 2011 IEEE International Conference on Systems, Man, and Cybernetics, Anchorage, AK, 2011, pp. 1037-1043. doi: 10.1109/ICSMC.2011.6083811

[15] Y. Saito and P. Raksincharoensak, "Shared Control in Risk Predictive Braking Maneuver for Preventing Collisions With Pedestrians," in IEEE Transactions on Intelligent Vehicles, vol. 1, no. 4, pp. 314-324, Dec. 2016. doi: 10.1109/TIV.2017.2700210

[16] S. C. Jugade, A. C. Victorino, "Grid based Estimation of Decision Uncertainty of Autonomous Driving Systems using Belief Function theory," in IFAC Symposium on Control in Transportation Systems (CTS), 2018, Pages 261-266, ISSN 2405-8963, https://doi.org/10.1016/j.ifacol.2018.07.043.

[17] A. Rusinowska, "Axiomatic and Strategic Approaches to Bargaining Problems," Theory and Applications of Relational Structures as Knowledge Instruments, Springer Berlin Heidelberg, 2003, pp. 124146. 